\title{
Coordination of Care Could Improve: Canadian Results from the Commonwealth Fund International Health Policy Survey of Primary Care Physicians
}

Vanessa Sovran, Alison Ytsma, Liudmila Husak and Tracy Johnson

\begin{abstract}
Care coordination is a critical component of a strong primary care system. The Commonwealth Fund (CMWF) 2019 International Health Policy Survey of Primary Care Physicians polled physicians in 11 countries, allowing international and pan-Canadian comparisons of physicians' perspectives in this area. Canadian physicians indicated that there was room for improvement in coordinating care with those outside their practice, particularly specialists, homebased care providers and social services. Opportunities may arise in learning from higher-performing CMWF countries and in adopting new information technologies that are growing methods of facilitating communication across care settings.
\end{abstract}

\section{Introduction}

Primary care clinicians, as patients' first point of contact with the healthcare system, are a vital component of the overall health system. Countries with strong primary care have better health outcomes, lower mortality rates and lower healthcare costs (Starfield et al. 2005). The Commonwealth Fund (CMWF) 2019 International Health Policy Survey of Primary Care Physicians elicits the perspectives of primary care physicians in 11 countries, including Canada. The survey delivers international and pan-Canadian comparisons on topics such as access to primary care, providing patient-centred care, coordination of care and adoption of information technologies.

Good care coordination is widely recognized as a crucial part of primary care provision (Hofmarcher et al. 2007; McMurchy 2009). In Canada, the primary care landscape has been undergoing reform, with jurisdictions using differing approaches (Peckham et al. 2018). Against this dynamic backdrop, it is useful to examine what Canadian primary care physicians think about the current state of care coordination across the country. Survey responses highlight that primary care physicians, similar to their CMWF counterparts, have challenges in coordinating patient care with providers outside their practice (CIHI 2020).

\section{Methodology}

The CMWF 2019 International Health Policy Survey of Primary Care Physicians polled physicians in 11 countries: Australia, Canada, France, Germany, the Netherlands, New Zealand, Norway, Sweden, Switzerland, the UK and the USA. In Canada, there were 2,569 respondents and an overall response rate of $39.3 \%$.

The 2019 survey included Yukon, the Northwest Territories and Nunavut for the first time. Owing to small sample sizes, the results from all territories were reported together (with permission and support). As the territories altogether do not represent one jurisdiction, no statistical tests were performed to compare this grouping to the CMWF average.

Primary care physicians were randomly selected in most provinces. In Prince Edward Island and the territories, all primary care physicians were invited to participate. Physicians were contacted by mail and could respond via either a paper survey or an online link.

The Canadian Institute for Health Information (CIHI) applied statistical methods to determine whether Canadian and provincial results were significantly different from the international average of 11 countries.

\section{Key Findings on Coordination of Care}

The responses of Canadian primary care physicians illuminate challenges in communication and patient information exchange with sites and providers outside their practices. Although $90 \%$ of physicians send the patient history to the specialist (higher than the CMWF average of $85 \%$ ), only $17 \%$ of physicians receive timely results back from the specialist (lower than the CMWF average of $21 \%$ ). Patients have also noticed this communication gap: in a 2016 CMWF survey, $21 \%$ of Canadians reported that their primary care doctor did not seem informed and up-to-date on the specialist care they received (CIHI 2017).

Canadian physicians responded similarly to CMWF physicians regarding their communication with hospitals. About half of primary care physicians receive a notification about a patient's emergency department visits (49\%), similar to the CMWF 
average (51\%). The highest proportion was in New Brunswick, though there was a wide range across jurisdictions (from 17\% to $87 \%$ ). Also, about half of Canadian physicians receive a notice of a patient's hospital stay $(54 \%$, compared to the CMWF average of $55 \%$ ). Notably, a few countries achieved high proportions of physicians receiving notices that their patients were seen in the emergency department (New Zealand: 85\%, the Netherlands: $84 \%$ ) and that their patient was admitted to a hospital (the Netherlands: $82 \%$; New Zealand: 79\%).

In all, $24 \%$ of Canadian primary care physicians communicate with home-based nursing care providers about their patients' needs and services, which is less than the CMWF average of $31 \%$. Figure 1 illustrates the variation across the provinces and territories. Likewise, about a third of Canadian physicians (36\%) are advised by home-based nursing care providers about changes in their patients' condition or health status, similar to the CMWF average (37\%).

\section{FIGURE 1.}

For patients who receive home-based nursing care, the percentage of primary care physicians who usually communicate with home-based nursing care providers about their patients' needs and services

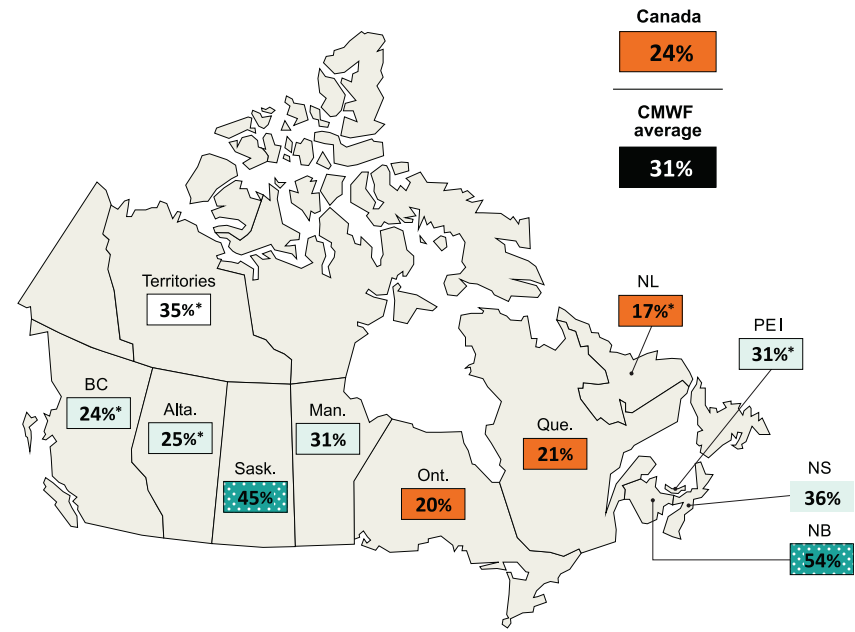

Above average $\bigcirc$ Same as average $\bigcirc$ Below average

Non-directional or no statistical tests were performed

to compare with the CMWF average (e.g., territories)

$\mathrm{CMWF}=$ Commonwealth Fund .

*The coefficient of variation is between $16.6 \%$ and $33.3 \%$; use with caution.

Note: This figure excludes respondents who answered "Does not apply." The colour code indicates statistical significance and the desirable direction of the indicator. The CMWF average was calculated by adding the results from the 11 countries and dividing by the number of countries. The Canadian average represents the average experience of Canadians (as opposed to the mean of provincial and territorial results).

Many Canadian physicians screen their patients for social needs $(60 \%)$, such as problems with housing, financial security and food insecurity. Fewer physicians frequently coordinate the care with social services (43\%), and this proportion has decreased since 2015 (from 50\%). Both of these are similar to responses from other CMWF physicians (the CMWF average for screening is $60 \%$ and for coordinating care is $46 \%$ ). Germany (at 75\%) and the UK (at 65\%) have higher proportions of doctors frequently coordinating care with social services than the CMWF average.

Canadian physicians reported various barriers when coordinating care with social services, with one of the top challenges being inadequate staffing to make referrals and coordinate care (Figure 2). Challenges ranked differently across the provinces/ territories.

\section{FIGURE 2.}

Proportion of primary care physicians who reported the following as major challenges when they or other personnel in their practice coordinated their patients' care with social services

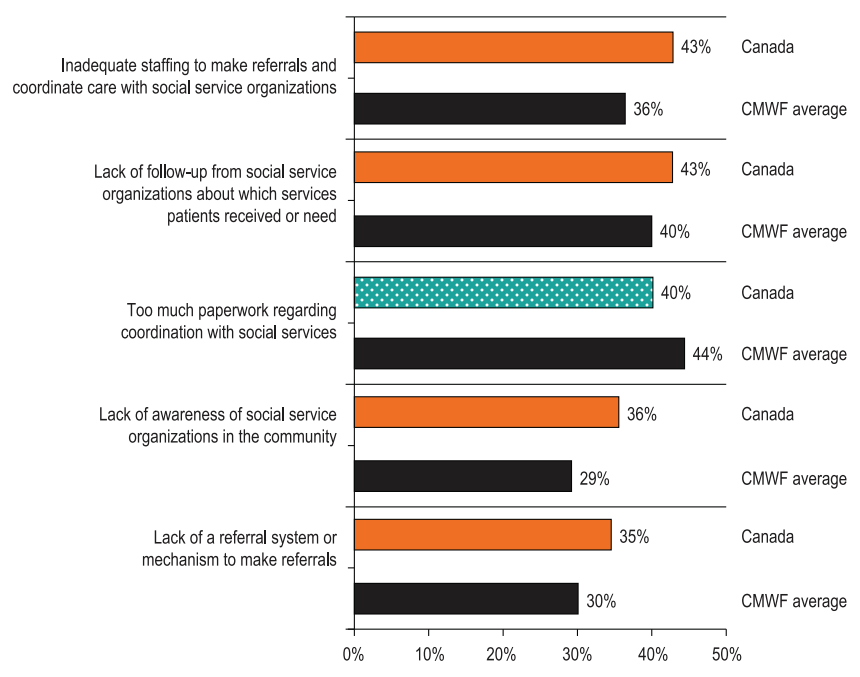

$\mathrm{CMWF}=$ Commonwealth Fund

Note: This figure excludes respondents who answered "Do not coordinate with social services." The colour code indicates statistical significance and the desirable direction of the indicator. Lower results are more desirable. The Commonwealth Fund average was calculated by adding the results from the 11 countries and dividing by the number of countries. The Canadian average represents the average experience of Canadians (as opposed to the mean of provincial and territorial results).

Although Canada has had more physicians using electronic medical records (EMRs) in $2019(86 \%)$ than in 2015 (73\%), fewer Canadian doctors are able to share patient information electronically with physicians outside their practice compared to their CMWF counterparts. While information sharing has increased in Canada and in other surveyed countries since 2015, fewer Canadian physicians exchange patient clinical summaries, laboratory and diagnostic test results and patient medication lists with doctors outside their practice than the CMWF average (Figure 3). Most provinces and territories were below the CMWF average in these areas, and none was above the average.

When surveyed on their perception of the health system's performance, $65 \%$ of Canadian physicians thought that better 


\section{FIGURE 3.}

Proportion of primary care physicians who can electronically exchange information with any doctor outside their practice

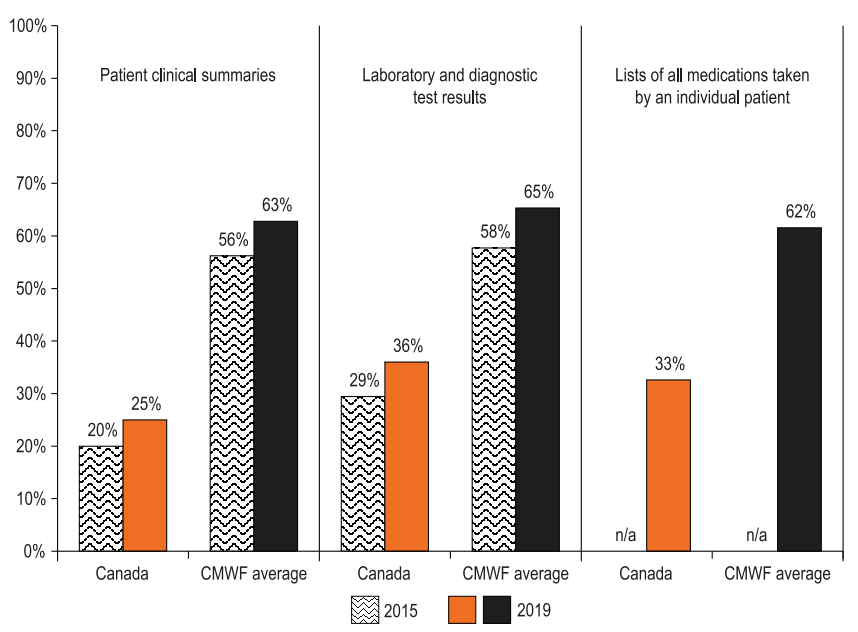

CMWF = Commonwealth Fund; $\mathrm{n} / \mathrm{a}=$ data are not available because this was a new question in 2019. Note: The colour code indicates statistical significance and the desirable direction of the indicator. The CMWF average was calculated by adding the results from the 11 countries and dividing by the number of countries. The Canadian average represents the average experience of Canadians (as opposed to the mean of provincial and territorial results).

integration of primary care with hospitals, mental health services and community-based social services is the top priority to improve quality of care and patient access.

\section{Conclusion}

The results of the CMWF 2019 International Health Policy Survey of Primary Care Physicians show that there is room for improvement in how Canadian physicians coordinate their patients' care. While the proportion of Canadian physicians receiving notifications about their patients' hospital interactions is similar to the CMWF average, it is still below the average in receiving timely information from specialists and in communicating with home-based care providers.

Canada's provinces and territories may be able to learn from higher performing countries. One approach, used by many CMWF physicians and increasingly adopted in Canada, is to utilize electronic technologies, such as EMRs, and other means of electronic communication with patients. If properly integrated with each other, these tools may facilitate information sharing and communication.

Most Canadian primary care physicians indicated that better integration of primary care with other sectors is the top priority, underlining the importance of making gains in this area. By improving their patients' care coordination, Canadian primary care physicians can further realize their potential as the hub where patients can receive safer, more effective and more integrated care.
Additional information that highlights the Canadian results can be found online at https://www.cihi.ca/en/ commonwealth-fund-survey-2019. Full data sets of the survey results are available to researchers upon request at cmwf@cihi.ca. HQ

\section{References}

Canadian Institute for Health Information (CIHI). 2017. How Canada Compares: Results from the Commonwealth Fund's 2016 International Health Policy Survey of Adults in 11 Countries. Retrieved April 22, 2020. <https://www.cihi.ca/sites/default/files/document/ commonwealth-fund-2016-chartbook-en-web-rev.pptx>.

Canadian Institute for Health Information (CIHI). 2020. How Canada Compares: Results from the Commonwealth Fund's 2019 International Health Policy Survey of Primary Care Physicians. Retrieved April 22, 2020. <https://www.cihi.ca/sites/default/files/document/cmwf-2019chartbook-en-web.pptx>.

Hofmarcher, M., H. Oxley and E. Rusticelli. 2007. Improved Health System Performance through Better Care Coordination. OECD Health Working Papers, No. 30. doi:10.1787/246446201766.

McMurchy, D. 2009, January. What Are the Critical Attributes and Benefits of a High-Quality Primary Healthcare System? Submitted to the Canadian Working Group on Primary Healthcare Improvement. Retrieved April 22, 2020. <https://www.cfhi-fcass.ca/Libraries/ Primary_Healthcare/11498_PHC_McMurchy_ENG_FINAL.sflb. ash $x>$.

Peckham, A., J. Ho and G. Marchildon. 2018, March. Policy Innovations in Primary Care across Canada. Retrieved April 22, 2020. <https:// ihpme.utoronto.ca/wp-content/uploads/2018/04/NAO-RapidReview-1_EN.pdf>.

Starfield, B., L. Shi and J. Macinko. 2005. Contribution of Primary Care to Health Systems and Health. Milbank Quarterly 83(3): 457-502. doi:10.1111/j.1468-0009.2005.00409.x.

\section{About the Authors}

Vanessa Sovran, HBSc, is a senior coordinator in health reports at $\mathrm{CIHI}$ in Toronto, ON. She supports analytic projects and is responsible for knowledge translation/exchange, stakeholder engagement and project management activities. She can be reached at vsovran@cihi.ca.

Alison Ytsma, BSc, is a program lead in health reports at $\mathrm{CIHI}$ in Toronto, ON. She leads a variety of analytic projects and has led a number of ClHI's international projects, including the Commonwealth Fund survey and the Organisation for Economic Co-operation and Development eTool project.

Liudmila Husak, MD, MPH, is a manager in health reports at $\mathrm{ClHI}$ in Toronto, ON. She is responsible for managing analytic projects and overseeing the work of the team.

Tracy Johnson, BScPT, MBA, is the director of health system analysis and emerging issues at $\mathrm{CIHI}$ in Toronto, ON. She is responsible for the vision, leadership and stakeholder relationships to develop analytical products. 